\title{
Correlation between decimetric radio emission and hard X-rays in solar flares
}

\author{
B. P. Da̧browski and A. O. Benz
}

\author{
Institute of Astronomy, ETH Zürich, Wolfgang-Pauli-Srasse 27, 8093 Zürich, Switzerland \\ e-mail: dabrowski@astro.phys.ethz.ch
}

Received 8 October 2008 / Accepted 31 March 2009

\section{ABSTRACT}

\begin{abstract}
Aims. The emission of decimetric flare radiation, in particular narrowband spikes and pulsations, is generally considered to originate in accelerated, non-thermal particles. On the other hand, non-thermal hard X-rays are also understood to be products of this acceleration. Do radio emission and hard X-ray signatures originate from the same acceleration process? A strong correlation between the light curves in the radio and HXR ranges may help answer this question.

Methods. The delay between the radio and hard X-ray emission was determined by cross-correlation. The time profiles of X-ray and radio emission include a wide range of energies and frequencies. Thus, correlation is not simply a yes/no question, but must be systematically searched for in various ranges. The high spectral resolution of RHESSI ensured that it was possible to carefully choose the energy range, excluding thermal emission. The broad bandwidth of Phoenix-2 allowed the selection of any emission in the full decimetre range. The energy range and duration in hard X-rays, and the frequency range in radio spectrograms were chosen to optimize the correlation. The cross-correlation coefficient was then analyzed by a Gaussian fitting method.

Results. The measured delays have a distribution of FWHM $4.9 \mathrm{~s}$ and $4.7 \mathrm{~s}$ for pulsations and spikes, respectively, evaluated from such a Gaussian fitting method. The mean delay for pulsations was found to be $-1.4 \pm 0.9 \mathrm{~s}$ (minus indicates that hard X-ray emission comes first), and for narrowband spikes to $-2.5 \pm 2.5 \mathrm{~s}$. There are broad wings in the distribution, which we interpret as chance coincidences. The delays do not depend on centre frequency, cross-correlation coefficient, duration of the correlating sequence, and position on the disk. However, we find an increase in the delay for the spikes with GOES magnitude (peak soft X-ray emission) of the flare and with peak hard X-ray flux. This was not the case for pulsations.

Conclusions. In contrast to previous reports, the average delays for all pulsations and all spike groups are consistent with zero. Thus, correlated decimetric pulsations and spikes are, on average, concomitant with non-thermal X-rays.
\end{abstract}

Key words. Sun: flares - Sun: corona - Sun: radio radiation - Sun: X-rays, gamma rays - acceleration of particles

\section{Introduction}

The intense emission of decimetric radiation from solar and stellar flares is generally considered to originate from non-thermal electron velocity distributions. These distributions can be unstable to maser emission or plasma waves that couple coherently with radio waves. Non-thermal velocity distributions originate directly from acceleration processes in flares, or may be driven to instability by particle trapping or propagation. The prototypical association of acceleration and radio emission is the correlation of narrowband spikes with type III bursts in metre waves (Benz et al. 1996), where the type III bursts are caused by propagating electron beams and the spikes are emitted close to or inside the acceleration region by some unknown process. Direct radio emission from the acceleration region may provide information about the mechanisms involved in this region.

On the other hand, hard X-rays (HXR) emitted by nonthermal electrons are known to be produced by this type of acceleration. If the radio and X-ray emitting electrons were accelerated by the same process, a correlation should exist between the two types of emission. In contrast, radio emission correlating with HXR possibly carry complementary information about acceleration or particle propagation and trapping. Benz et al. (2005) showed that about every HXR flare is associated with coherent radio emission, where associated means that the radio event occurs during the enhanced HXR emission. However, for a correlation to exist, we require that both of the light curves, radio and $\mathrm{X}$-ray emission, evolve at the same time. Occasional strong correlation has been reported between type III bursts and X-rays (Kane 1972; Kane 1981; Kane et al. 1982; Aschwanden et al. 1995; Arzner \& Benz 2005). Here, we concentrate on radio emission that is not classified as type III bursts, thus is presumably not emitted by propagating electron beams. Coherent radio emission at decimetre wavelengths originates in either a plasma density between $3 \times 10^{8}$ and $3 \times 10^{10} \mathrm{~cm}^{-3}$ or a magnetic field between 100 and $1000 \mathrm{G}$, assuming second harmonic emission is present. Both ranges cover values typically expected for flare acceleration regions.

Among the different types of coherent emission in the decimetre range, narrowband spikes are of greatest interest as possible radiations from close or within the acceleration region in analogy to the radio emission in the metre wavelength band. A close correlation between narrowband spikes in the decimetre range and HXR was first reported by Benz (1985) and Benz \& Kane (1986). Since the peak flux of individual spikes does not vary significantly in the course of a flare, the number of spikes also correlates with the HXR flux (Benz 1985; Aschwanden \& Güdel 1992). Around $95 \%$ of the radio events with narrowband decimetric spikes are associated with HXR emission (Güdel et al. 1991). The time profile integrated over the band, in which spikes were observed, and the time profile of HXR were found to have a high cross-correlation coefficient 
(Güdel et al. 1991; Aschwanden \& Güdel 1992; Fárník \& Karlický 2005). However, Güdel et al. (1991) noted that the $\mathrm{X}$-rays are observed $4 \pm 9 \mathrm{~s}$ earlier than the radio emission. Aschwanden \& Güdel (1992) reported that the spikes appear later by between 2 and $5 \mathrm{~s}$, the greater the HXR flux, the later the radio emission. However, Fárnik \& Karlický (2005) found no similar correlation for a smaller sample. The reported delay of decimetric spikes was generally interpreted as implying that the spikes are a secondary effect caused by propagation and/or trapping (review by Fleishman \& Mel'nikov 1998). On the other hand, Fleishman et al. (2003) correlated radio spikes in the $1.2-3.4 \mathrm{GHz}$ frequency range with gyrosynchrotron emission at $6.6 \mathrm{GHz}$. On average, they found that the spike emission arrives $5.1 \mathrm{~s}$ earlier. There is a general delay between the centimetric emission and the HXR of on average $0.2 \mathrm{~s}$ at $10.6 \mathrm{GHz}$ (Cornell et al. 1984). At low time resolutions, which emphasize longer time scales, the delay in the centimetre emission is typically 2 s (Starr et al. 1988; Lu \& Petrosian 1990).

Narrowband spikes are relatively rare tracers of acceleration. Among all solar flares observed in soft X-rays (SXR) by GOES (Da̧browski et al. 2005) and in HXR by HRBS/SMM (Aschwanden \& Güdel 1992), only 2\% of them are associated with decimetric spikes. The number increases to $14 \%$ for spikes originating in flares observed in HXR by the RHESSI satellite (Benz et al. 2005).

For quasi-periodic, broadband decimetric pulsations, the association rate is $80 \%$ (Benz et al. 2005). Decimetric pulsations have various shapes and structures (Güdel \& Benz 1988; Isliker \& Benz 1994; Jiřička et al. 2002). Some have short duration elements comparable to those of spikes. The first systematic research on the association of decimetric pulsations with HXR was conducted by Aschwanden et al. (1990). They also found occasional correlations. Investigating the correlation in more detail, Kliem et al. (2000) reported an anticorrelation between the fine structures in pulsating radio emission and HXR. This anticorrelation could also be interpreted as a phase shift or delay of $1.3 \mathrm{~s}$. Arzner \& Benz (2005) reported a delay of HXR relative to decimetric pulsations between $-6 \mathrm{~s}$ and $0.46 \mathrm{~s}$. The mean delay was $-1.1 \mathrm{~s}$ (HXR first) and did not differ significantly from zero. We finally note that pulsations appear both in the rise phase of SXR (impulsive phase of flare) as well as in the decay phase of SXR (Magdalenić et al. 2002).

Here, we present an investigation of the relation between coherent decimetric emission and HXR. The radio emission was observed by Phoenix-2 (Benz et al. 1991; Messmer et al. 1999). For the first time, we have the possibility of a systematic investigation of the radio-HXR relation for the range from 300 to 3000 MHz. HXRs were observed by RHESSI (Lin et al. 2002). The high spectral resolution of RHESSI made it possible to both carefully choose the energy range and maximize the non-thermal counts, excluding thermal emission. We use the method developed by Arzner \& Benz (2005) and apply it to the full set of available data, focusing on decimetric spikes and pulsations.

\section{Observations}

Phoenix-2 is a broadband radio spectrometer located at Bleien, Switzerland $\left(8^{\circ} 6^{\prime} 44^{\prime \prime} \mathrm{E}, 47^{\circ} 20^{\prime} 26^{\prime \prime} \mathrm{N}\right)$. The antenna has a parabolic $7 \mathrm{~m}$ dish. The frequency-agile spectrometer measures the total flux density and the circular polarization (bandwidth: 1,3 or $10 \mathrm{MHz}$ ). It records the full Sun radio emission between $100 \mathrm{MHz}$ and $4 \mathrm{GHz}$ from sunrise to sunset. During the time of the selected observations, the 2000 measurements available per second were distributed into 200 frequency channels, yielding a
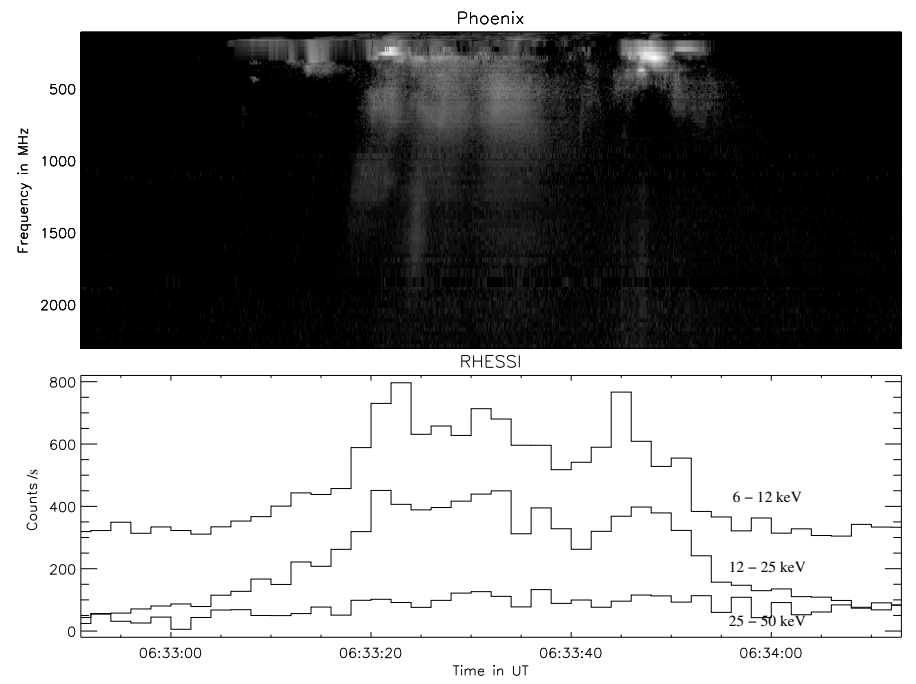

Fig. 1. The event of August 14, 2004 observed by the Phoenix-2 radio spectrometer (top) and the RHESSI X-ray satellite (bottom). The event was selected for detailed analysis. The radio spectrum was cleaned from interference and shows a decimetric pulsation. The X-ray counts were binned to $2 \mathrm{~s}$ in time and to 3 energy channels.

time resolution of $0.1 \mathrm{~s}$ in each channel. The radio data were calibrated and cleaned from terrestrial interference using standard routines. The radio flux density was correlated with RHESSI data. The polarimetric data were used only for additional help in the classification of the solar radio events, in particular for identifying and eliminating type III bursts and gyrosnchrotron radiation that are generally weakly polarized. The radio spectrogram is integrated over a finite bandwidth to obtain a single time profile. The broad bandwidth of Phoenix-2 allows us to select any emission in the full decimetre range. An example of the data is presented in Fig. 1.

The Reuven Ramaty High Energy Solar Spectroscopic Imager (RHESSI) satellite observes X-rays in the range from $3 \mathrm{keV}$ to $17 \mathrm{MeV}$. The germanium detectors register photons with an energy resolution of about $1 \mathrm{keV}$. The high spectral resolution allows us to differentiate well between thermal and non-thermal emissions and to carefully select the energy range of non-thermal photons to be correlated. The imaging technique requires the satellite to rotate with a period of about $4 \mathrm{~s}$, modulating the signal. To allow for higher time resolution, the time profile must be demodulated. Using the information for the satellite pointing and the X-ray source position on the solar disk, the modulation can be calculated and eliminated. We use a visibilitybased method by Arzner (2004).

The accuracy of the timing of the two instruments is essential to the study of temporal correlation, because time delays may have instrumental origins. In Phoenix-2, the time stamp is given by GPS. The timing is delayed by two instrumental effects: $(i)$ the delay of the solar signal by internal electronics, which amounts to $22 \pm 2 \mathrm{~ms}$; (ii) the time of the frequency-agile spectrometer refers to the first channel. Thus, the timing of the $n$th channel is shifted by $(n-1) \times 0.5 \mathrm{~ms}$, which is on average by $50 \mathrm{~ms}$. The RHESSI clock is also locked to GPS, and its time stamp is set to within approximately $1 \mu \mathrm{s}$. A systematic effect arises from the different light travel times to Phoenix-2 and RHESSI. The maximum possible delay is given by the radius of the Earth, and is thus $20 \mathrm{~ms}$. The radio signal is thus systematically delayed by $0.072 \pm 0.07 \mathrm{~s}$. The mean delay has been corrected in the following where relevant. 
Solar effects may cause far longer delays. Of particular importance are the reduced propagation speed of radio waves in the coronal plasma and the possibly greater distance of the origin of $\mathrm{X}$-rays emitted from the footpoints. These effects are discussed later.

\section{Data selection}

Since there are decimetric pulsations without enhanced X-ray emission and X-ray flare emission without decimetric pulsations or spikes, the selection is very important. There may thus be radio and X-ray emission without direct relation to each other. The selection must exclude chance coincidences on the one hand, but include directly connected emission with considerable delays. The new data allowed not only a selection of events, but a more effective search for correlations between emission in the $\mathrm{X}$-ray energy range and radio frequency range than in previous investigations.

The selection was made from flare radio emissions classified as "decimetric spikes, pulsations or continua" (abbreviated as DCIM) from the Phoenix-2 burst list published in Solar and Geophysical Data. The time interval covered February 5, 2002 (date launch of RHESSI satellite) until the end 2007. Out of the initial 870 DCIM events, 425 were observed simultaneously with RHESSI. The events were inspected in quicklook images. Continua that increased toward higher frequencies beyond $4 \mathrm{GHz}$ were excluded as possible gyro-synchrotron emissions. Furthermore, we required a temporal coverage by RHESSI of at least $75 \%$. This reduced our sample to 169 events. Out of these, 107 radio events were of the decimetric pulsation type and in 32 events we found narrowband spikes.

In addition to entire events, we also studied isolated groups of pulsations or spikes within an event. Every group was analyzed individually. Because of the large number and often long duration of observed pulsations, for detailed analysis we chose only groups well separated from other emission. Since spikes are not as frequent, we studied all events, but distinguished well isolated groups of spikes from spikes that were mixed with other types of radio emission. Groups of spikes with only a few spikes were excluded. Furthermore we omitted,

- groups of pulsations and spikes during which the attenuator on RHESSI satellite was changed;

- groups during which problems with the positioning of the RHESSI satellite occurred;

- groups during which RHESSI went into eclipse or South Atlantic Anomaly;

- long duration events beyond 15 minutes;

- groups with unavailable source positions, which is needed for demodulation.

By excluding groups and events with poor data, we finally obtained 102 groups (50 radio events) of pulsations and 25 groups (19 radio events) of spikes with good RHESSI coverage.

The final step was the most difficult. From the HXR covered groups and events, we had to exclude those that appeared not to be correlated, i.e., their time profiles obviously differed from the HXR light curve. Next we applied a cross-correlation with HXR for a total of 37 groups (28 radio events) of pulsations and 12 groups (10 radio events) of spikes with the method described below. From this selection, we excluded events if the peak of the cross-correlation function between radio and HXR was delayed by more than $20 \mathrm{~s}$ and $-20 \mathrm{~s}$ to reduce the risk of chance coincidences. This limit is justified later from the distribution of the
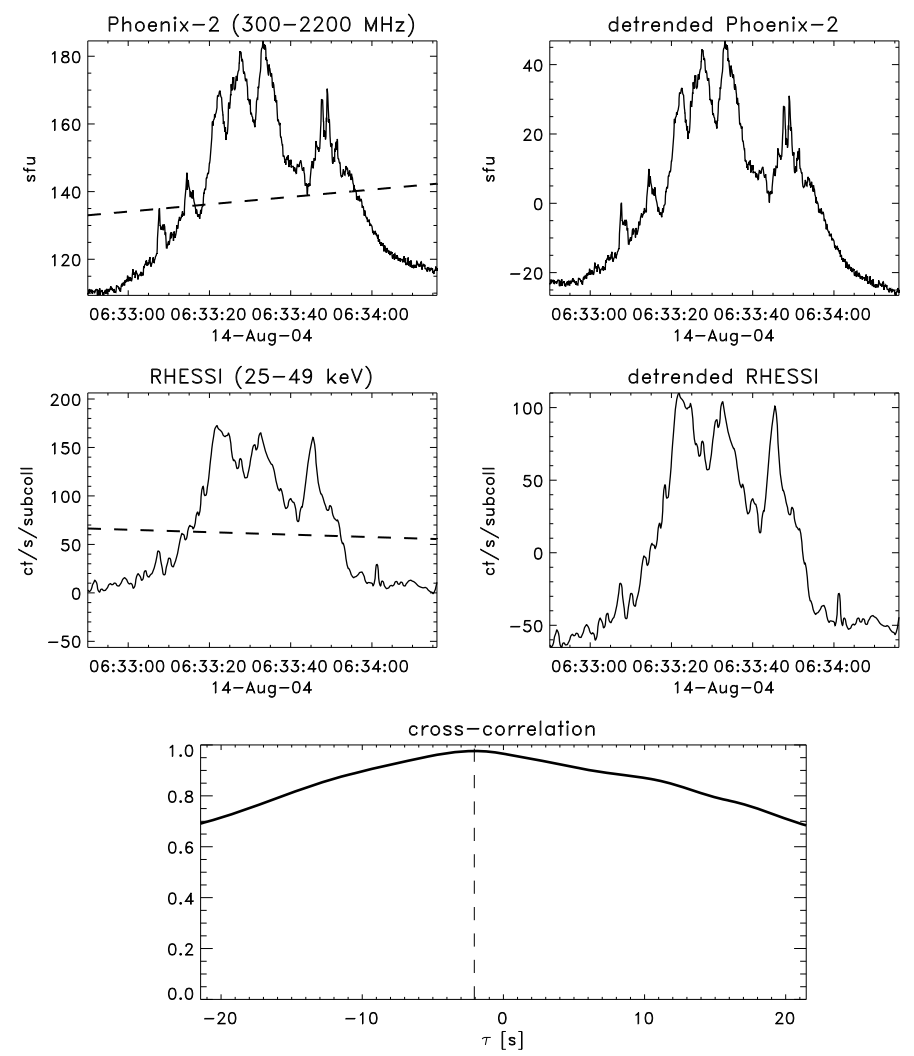

Fig. 2. Time profiles of Phoenix-2 observations (top row) and RHESSI observations (middle row) during the event of Fig. 1. The frequency and energy ranges, respectively, are given at the top of each figure. A linear trend (left column, dashed) is subtracted to obtain de-trended light curves (right column). The cross-correlation coefficient between radio light curve and hard X-rays curve is shown in the bottom panel. The units are seconds, a positive shift meaning that X-rays are delayed.

observed delays showing a bell-shaped kernel and a flat background. Finally, 33 groups (26 radio events) of pulsations and 11 groups (9 radio events) of spikes remained in the selection for the detailed analysis.

The selection reflects the number of events that are well correlated with HXR: $32 \pm 6 \%$ of the groups (52 $\pm 10 \%$ of the events) of decimetric pulsations, and $44 \pm 24 \%$ of the groups $(47 \pm 29 \%$ of the events) of all narrowband spikes.

\section{Radio-HXR correlation and data analysis}

The delay between decimetric radio emission and HXR is determined by cross-correlation. To increase the accuracy, the RHESSI signal is demodulated before cross-correlation, using the code described in Arzner \& Benz (2005).

In the first step, we select a time interval and an energy range from a spectrogram of HXR emission. The range is restricted to non-thermal emission, which usually determines the lower limit to the range, and defined to avoid background noise, which determines the upper limit. Secondly, a time-frequency range is chosen from the radio spectrogram to select the pertinent radio emission. In the third step, the selected dynamic radio spectrum is integrated with frequency to obtain a light curve. The HXR spectrum from the selected box is integrated with energy and binned to the time resolution of the radio data $(0.1 \mathrm{~s})$ to obtain the HXR light curve. The linear trends are then subtracted from both curves, by assuming that the trend is similar to a background and independent of the modulation (see Fig. 2-top and middle row). 
In the end, the cross-correlation between radio light curve and HXR curve is carried out and the cross-correlation coefficient is plotted versus time shift (Fig. 2 - bottom panel).

The delay is defined as the time difference between the radio signal and HXR. It is defined to be positive if the radio emission precedes. There are two definitions of the delay:

- the time shift of the peak of the cross-correlation function. This will be referred to as the "maximum method" in the following;

- since the cross-correlation coefficient sometimes has a rather flat peak, the delay measurement can be improved by a Gaussian function fit to the cross-correlation function ("Gauss method").

For both the maximum method and the Gauss method, the peak value of the cross-correlation function was also determined to quantify the quality of the correlation. In the following, it is simply referred to as correlation coefficient.

To determine possible relations between the delay and other parameters, we use linear regression analysis. The output variables (regression coefficient and standard deviation) are computed in the following 3 ways:

- ordinary least-squares regression $y$ versus $x$;

- ordinary least-squares regression $x$ versus $y$;

- bisector between the two.

Since all parameters are measured quantities, we use all three methods to test the robustness of the result. As a reference value for final results, we refer to the bisector.

\section{Results}

We present a temporal comparison between the radio and HXR emission during spikes and pulsations, which were selected for the purpose of identifying possible correlations.

\subsection{Delays}

The selected groups of pulsations and cross-correlation results are presented in Table 1 . Table 2 presents the same for narrowband decimetric spikes. The difference between the delays determined by the Gauss method and the maximum method is of the order of seconds. Some of the largest differences, such as those of the events 25.10.2004 (pulsations) and 11.06.2003 (spikes), occur for low correlation coefficients. Tables 1 and 2 also give the mean values of the soft X-ray flux observed by GOES and the HXR flux observed by RHESSI in the correlated interval. They indicate the flare class and HXR count statistics to be used later in the regression analysis.

Negative delays mean that HXR emission precedes radio emission. We note that there are both positive and negative delays. In the majority, the delays determined by the maximum and Gauss methods have the same sign. Using the Gauss method, 18 groups of $33(55 \%)$ with pulsating decimetric emission show a negative delay. The number of spike groups is smaller, but the results are very similar: 7 groups of $11(64 \%)$ have a negative delay. For both pulsations and spikes, the differences between Gauss method and maximum method are insignificant. We note that the slightly higher number of negative delays is statistically insignificant. Positive and negative delays appear to be equally likely.

The mean delays of pulsations and spikes are presented in Table 3. The table summarizes the averages over all groups listed in Tables 1 and 2. The standard error of the mean value is given by the standard deviation divided by the square root of the number of samples (Poisson statistics) for pulsations. Since the number of spike groups is lower than 30, the error was corrected using Student's-t statistics. The correction number amounts to a factor of 1.812 according to 10 degrees of freedom and a statistical reliability $\alpha=0.9$. For both pulsations and spikes, the Gauss and maximum methods agree to within the standard error of the mean, although for individual cases the two methods may deviate considerably as noticed in Tables 1 and 2 .

The distribution of the delays is broad. The standard deviation evaluated for all groups in Tables 1 and 2 equals $4.9 \mathrm{~s}$ and $4.0 \mathrm{~s}$ for pulsations using the Gauss and maximum methods, and $4.7 \mathrm{~s}$ and $4.5 \mathrm{~s}$ for spikes from the Gauss and maximum methods.

Figures 3 and 4 present the delay distributions for pulsations and spikes. A Gaussian was fitted into the histogram, using a bin width of $1 \mathrm{~s}$, and is also shown in the figures by the solid curve. This fitting of the delay distribution yields an alternative estimate of the $F W H M$, the mean delay, and its error. The results are given in Table 3.

The fitting weights the result towards the kernel of the distribution around zero delay. For the standard deviation analysis, the wings have more weight. Thus, the difference suggests that the distribution is not Gaussian, but consists of a kernel around zero delay and broad wings.

We conclude from the various statistics of the delay signs and the mean value of the delays that individual pulsation and spike groups may have delays of several seconds in the positive and negative direction. We note that in both types of emission there are wings in the distribution of delays of up to $\pm 10 \mathrm{~s}$ and more. There is a trend for negative delays (HXR first), which produce average delays of about $-1 \mathrm{~s}$ (see second line in Table 3 ). However, this trend is statistically insignificant and not robust. If the mean is marginally different from zero for one method, it is not in the other method. Thus, we conclude that the average delays for both pulsations and spikes in our data are consistent with zero.

\subsection{Correlation coefficient}

The correlation coefficient for pulsations evaluated by the Gauss method is between 0.18 and 0.96 , and for the maximum method it is between 0.18 and 0.98 . The mean correlation coefficient for pulsations evaluated by the Gauss method is 0.7 and by the maximum method 0.8 . The correlation coefficient for spikes evaluated by the Gauss method is between 0.32 and 0.90 , and for the maximum method is between 0.32 and 0.95 . For spikes, the mean correlation coefficient evaluated by both the Gauss and maximum method is found to be 0.7 .

The correlation between decimetric radio emission and HXR is not excellent, but statistically highly significant for individual events.

\subsection{Relations of delay to other parameters}

In Figs. 5 and 6, the 33 pulsation groups are displayed as $\mathrm{X}$-ray flux versus delay. Pulsations are symmetrically distributed in terms delay with no obvious correlation with X-ray flux. However, they show a trend of having a lower absolute delay with higher HXR flux. Figures 7 and 8 display the 11 spikes groups. They are not symmetrical in terms of X-ray flux, but the 
Table 1. Analyzed groups of decimetric pulsations.

\begin{tabular}{|c|c|c|c|c|c|c|c|c|c|c|c|}
\hline $\begin{array}{c}\text { Date } \\
\text { [dd.mm.yy] }\end{array}$ & $\begin{array}{c}\text { Start time } \\
\text { [hh:mm:ss] }\end{array}$ & $\begin{array}{l}\text { End time } \\
\text { [hh:mm:ss] }\end{array}$ & $\begin{array}{c}\operatorname{pos}^{a} \\
{\left[{ }^{\prime \prime}\right]}\end{array}$ & $\begin{array}{c}E \_l^{b} \\
{[\mathrm{keV}]}\end{array}$ & $\begin{array}{l}v^{c} \\
{[\mathrm{MHz}]}\end{array}$ & cc_G $G^{d}$ & $\begin{array}{r}\Delta t_{-} G^{e} \\
{[\mathrm{~s}]}\end{array}$ & cc_max ${ }^{f}$ & $\begin{array}{r}\Delta t \_\max ^{g} \\
{[\mathrm{~s}]} \\
\end{array}$ & $\begin{array}{c}\phi_{-} G O E S^{h} \\
{\left[\mathrm{~W} \mathrm{~m}^{-2}\right]}\end{array}$ & $\begin{array}{r}\phi_{-} R H E S S I^{i} \\
{[\mathrm{ct} / \mathrm{s} / \mathrm{subcoll]}]}\end{array}$ \\
\hline 05.12 .06 & $11: 15: 06$ & 11:18:07 & 974 & 16 & $300-847$ & 0.72 & 4.07 & 0.80 & 8.07 & $4.05 \times 10^{-5}$ & 261.7 \\
\hline 20.08 .06 & 07:08:52 & $07: 12: 58$ & 909 & 20 & $1180-2950$ & 0.50 & 11.69 & 0.53 & 8.97 & $3.61 \times 10^{-7}$ & 25.2 \\
\hline 04.08 .05 & $17: 36: 18$ & $17: 37: 55$ & 423 & 25 & $336-995$ & 0.60 & -11.65 & 0.65 & -7.18 & $2.49 \times 10^{-7}$ & 15.7 \\
\hline 01.08 .05 & $16: 26: 50$ & $16: 28: 32$ & 568 & 7 & $309-542$ & 0.35 & -5.45 & 0.39 & -4.38 & $6.35 \times 10^{-7}$ & 72.6 \\
\hline 14.07 .05 & $05: 24: 08$ & $05: 26: 04$ & 950 & 10 & $318-2650$ & 0.77 & -8.72 & 0.78 & -7.35 & $1.74 \times 10^{-6}$ & 200.3 \\
\hline 12.07 .05 & 08:08:21 & 08:08:45 & 822 & 17 & $515-1865$ & 0.74 & -0.32 & 0.74 & -1.21 & $6.77 \times 10^{-6}$ & 165.3 \\
\hline 15.05 .05 & $09: 20: 47$ & $09: 21: 23$ & 499 & 16 & $904-1660$ & 0.82 & 0.74 & 0.86 & -1.81 & $3.80 \times 10^{-7}$ & 50.0 \\
\hline 08.05 .05 & 12:08:14 & $12: 10: 02$ & 198 & 6 & $363-2470$ & 0.65 & -0.25 & 0.76 & -1.73 & $2.87 \times 10^{-7}$ & 46.8 \\
\hline 14.01 .05 & $11: 36: 17$ & $11: 37: 55$ & 395 & 15 & $336-1$ & 0.83 & -2.96 & & -7.24 & $1.34 \times 10^{-6}$ & 114.6 \\
\hline 13.01 .05 & $13: 06: 56$ & $13: 09: 11$ & 300 & 10 & $309-$ & 0.88 & 1.40 & & -1.52 & $6.62 \times 10^{-7}$ & 192.7 \\
\hline 25.10 .04 & $14: 40: 50$ & $14: 44: 56$ & 88 & 14 & $524-1300$ & 0.29 & 7.83 & & 2.56 & $4.43 \times 10^{-6}$ & 104.0 \\
\hline 20.09.04 & $07: 13: 45$ & 07:14:17 & 834 & 20 & $461-2110$ & 0.77 & -3.66 & & -3.51 & $4.88 \times 10^{-7}$ & 77.1 \\
\hline 14.08 .04 & $06: 32: 50$ & $06: 34: 16$ & 549 & 25 & 200 & 0.96 & -0.46 & & -1.96 & $9.89 \times 10^{-6}$ & 60.9 \\
\hline 11.08 .04 & 11:06:37 & $11: 0$ & 391 & 11 & $309-$ & 0.92 & 0.46 & & 1.82 & $1.98 \times 10^{-6}$ & 163.4 \\
\hline 11.08 .04 & $11: 06: 35$ & 11:08:12 & 391 & 11 & $533-1690$ & 0.85 & -9.48 & & -9.66 & $1.98 \times 10^{-6}$ & 162.0 \\
\hline 11.06 .03 & $17: 35: 26$ & $17: 37: 51$ & 462 & 15 & $300-989$ & 0.73 & -1.99 & & -3.67 & 1.65 & 64.9 \\
\hline 10.06 .03 & 11:06:00 & $11: 06: 44$ & 683 & 15 & $1210-2950$ & 0.96 & 1.36 & 0.97 & 1.53 & 1.90 & 570.1 \\
\hline 10.06 .03 & $11: 06: 43$ & $11: 07: 25$ & 683 & 15 & $1210-2950$ & 0.95 & 0.00 & 0.98 & -0.36 & $2.00 \times 10^{-5}$ & 698.8 \\
\hline 10.06 .03 & $14: 07: 32$ & $14: 09: 03$ & 707 & 25 & $327-1030$ & 0.79 & -2.43 & 0.92 & -1.89 & $8.70 \times 10^{-6}$ & 19.5 \\
\hline 25.11 .02 & $10: 41: 41$ & $10: 42: 19$ & 783 & 20 & $912-2950$ & 0.83 & 0.08 & 0.86 & -0.36 & $1.09 \times 10^{-6}$ & 334.7 \\
\hline 18.11 .02 & $12: 06: 08$ & $12: 06: 40$ & 959 & 20 & $452-1690$ & 0.69 & -3.13 & 0.73 & -1.74 & $1.72 \times 10^{-6}$ & 9.8 \\
\hline 31.10 .02 & $09: 25: 32$ & $09: 25: 53$ & 618 & 24 & $461-766$ & 0.61 & 0.54 & 0.64 & 0.61 & $1.11 \times 10^{-5}$ & 176.8 \\
\hline 20.10 .02 & $14: 22: 28$ & $14: 22: 53$ & 532 & 25 & $605-1030$ & 0.71 & -0.80 & 0.75 & -0.83 & $5.34 \times 10^{-6}$ & 151.5 \\
\hline 20.10 .02 & $14: 23: 27$ & $14: 24: 31$ & 532 & 25 & $417-1510$ & 0.18 & 0.33 & 0.18 & 0.42 & $9.09 \times 10^{-6}$ & 145.7 \\
\hline 20.10 .02 & $14: 25: 10$ & $14: 25: 58$ & 532 & 25 & $426-676$ & 0.49 & 2.30 & 0.53 & 0.39 & $1.33 \times 10^{-5}$ & 86.3 \\
\hline 20.10 .02 & $14: 26: 13$ & $14: 27: 25$ & 532 & 25 & $309-2020$ & 0.88 & -1.36 & 0.93 & -1.32 & $1.65 \times 10^{-5}$ & 184.5 \\
\hline 30.07 .02 & $17: 36: 42$ & $17: 38: 27$ & 243 & 25 & $309-667$ & 0.85 & -6.69 & 0.90 & -7.65 & $2.28 \times 10^{-6}$ & 50.6 \\
\hline 30.07 .02 & $17: 36: 42$ & $17: 38: 27$ & 243 & 25 & $659-1450$ & 0.85 & -3.04 & 0.91 & -4.08 & $2.28 \times 10^{-6}$ & 50.7 \\
\hline 07.02 & $17: 36: 42$ & $17: 38: 27$ & 243 & 25 & $1415-2950$ & 0.92 & -2.60 & 0.96 & -4.61 & $2.28 \times 10^{-6}$ & 50.6 \\
\hline 04.07 .02 & $17: 44: 36$ & $17: 45: 41$ & 914 & 21 & $766-1510$ & 0.49 & -13.29 & 0.52 & -6.51 & $1.74 \times 10^{-6}$ & 50.8 \\
\hline 03.06 .02 & $14: 43: 17$ & $14: 43: 40$ & 924 & 33 & $632-2950$ & 0.83 & 0.08 & 0.9 & 0.21 & $3.72 \times 10^{-6}$ & 52.4 \\
\hline 13.05 .02 & $07: 45: 12$ & $07: 45: 49$ & 329 & 8 & $1690-2950$ & 0.82 & 0.77 & 0.87 & 0.25 & $8.87 \times 10^{-7}$ & 36.4 \\
\hline 11.04 .02 & $16: 19: 45$ & $16: 22: 28$ & 538 & 25 & $1415-2950$ & 0.93 & 0.52 & 0.97 & 0.10 & $3.75 \times 10^{-6}$ & 45.3 \\
\hline
\end{tabular}

${ }^{a}$ Position from the centre of the solar disc; ${ }^{b}$ photon low-energy limit; ${ }^{c}$ frequency band; ${ }^{d}$ correlation coefficient for Gauss method; ${ }^{e}$ delay for Gauss method; ${ }^{f}$ correlation coefficient for maximum method; ${ }^{g}$ delay for maximum method; ${ }^{h}$ GOES $(1-8 \AA$ A $)$ soft X-ray flux; ${ }^{i}$ RHESSI hard $\mathrm{X}$-ray flux in the analyzed photon energy range.

Table 2. Analyzed groups of decimetric spikes.

\begin{tabular}{|c|c|c|c|c|c|c|c|c|c|c|c|}
\hline $\begin{array}{c}\text { Date } \\
\text { [dd.mm.yy] }\end{array}$ & $\begin{array}{c}\text { Start time } \\
\text { [hh:mm:ss] }\end{array}$ & $\begin{array}{c}\text { End time } \\
\text { [hh:mm:ss] }\end{array}$ & $\begin{array}{c}\operatorname{pos}^{a} \\
{\left[{ }^{\prime \prime}\right]}\end{array}$ & $\begin{array}{c}E \_l^{b} \\
{[\mathrm{keV}]}\end{array}$ & $\begin{array}{l}v^{c} \\
{[\mathrm{MHz}]}\end{array}$ & cc_G $G^{d}$ & $\begin{array}{r}\Delta t_{-} G^{e} \\
{[\mathrm{~s}]}\end{array}$ & cc_max ${ }^{f}$ & $\begin{array}{r}\Delta t \_\max ^{g} \\
{[\mathrm{~s}]}\end{array}$ & $\begin{array}{c}\phi_{-} G O E S^{h} \\
{\left[\mathrm{~W} \mathrm{~m}^{-2}\right]}\end{array}$ & $\begin{array}{r}\phi_{-} R H E S S I^{i} \\
\text { [ct/s/subcoll] }\end{array}$ \\
\hline 14.08 .04 & $12: 03: 53$ & $12: 05: 25$ & 590 & 20 & $1865-2950$ & 0.69 & 1.32 & 0.77 & -1.00 & $4.78 \times 10^{-6}$ & 44.4 \\
\hline 19.01 .04 & $12: 33: 46$ & $12: 34: 21$ & 193 & 25 & $685-1300$ & 0.70 & 0.65 & 0.74 & 0.73 & $4.70 \times 10^{-6}$ & 224.1 \\
\hline 10.06 .03 & 11:08:27 & 11:09:19 & 683 & 19 & $650-1570$ & 0.81 & -2.40 & 0.82 & -2.70 & $2.19 \times 10^{-5}$ & 122.5 \\
\hline 09.01 .03 & $13: 20: 31$ & $13: 21: 14$ & 850 & 25 & $515-904$ & 0.63 & 0.85 & 0.65 & 1.15 & $1.51 \times 10^{-6}$ & 13.6 \\
\hline 20.12 .02 & $14: 19: 04$ & $14: 19: 55$ & 623 & 15 & $983-1845$ & 0.90 & -1.71 & 0.95 & -1.70 & $1.98 \times 10^{-6}$ & 33.8 \\
\hline 20.09 .02 & $12: 37: 16$ & $12: 40: 52$ & 444 & 10 & $632-1540$ & 0.65 & 2.06 & 0.68 & 1.61 & $1.02 \times 10^{-6}$ & 25.5 \\
\hline 29.07 .02 & $11: 02: 14$ & $11: 03: 46$ & 371 & 18 & $632-1415$ & 0.64 & -10.64 & 0.66 & -12.56 & $3.59 \times 10^{-5}$ & 270.8 \\
\hline 29.07 .02 & $11: 05: 53$ & $11: 07: 23$ & 371 & 18 & $623-1090$ & 0.75 & -11.95 & 0.78 & -8.83 & $3.23 \times 10^{-5}$ & 213.6 \\
\hline 15.07 .02 & $11: 44: 24$ & $11: 44: 50$ & 229 & 25 & $542-721$ & 0.88 & -0.57 & 0.88 & 0.07 & $3.82 \times 10^{-6}$ & 242.9 \\
\hline $11.06 .03^{*}$ & $16: 27: 29$ & $16: 29: 56$ & 849 & 25 & $381-784$ & 0.32 & -4.55 & 0.32 & -0.49 & $3.60 \times 10^{-5}$ & 154.7 \\
\hline $15.07 .02 *$ & $11: 44: 25$ & $11: 45: 05$ & 229 & 25 & $721-1210$ & 0.76 & -0.58 & 0.78 & 0.10 & $3.99 \times 10^{-6}$ & 190.8 \\
\hline
\end{tabular}

higher the X-ray flux, the longer is the delay. This trend, however, is dominated by 2 groups out of 11 .

To verify the visual impression, we use linear regression as described in Sect. 4. The results of the three methods of regression are similar. For simplicity, we report our results only for the bisector value in Table 4 . The significance of the statistical result can be seen most clearly in the ratio of slope coefficient $b$ to its standard deviation $\Delta b$. It is lower than or equal to a factor of 3 for pulsations, but higher than 3 for spikes. The results are:

- there is no relation between delay and X-ray flux for the pulsations;

- the delay decreases (more negative) with X-rays for spikes, thus the slope coefficients are negative. 

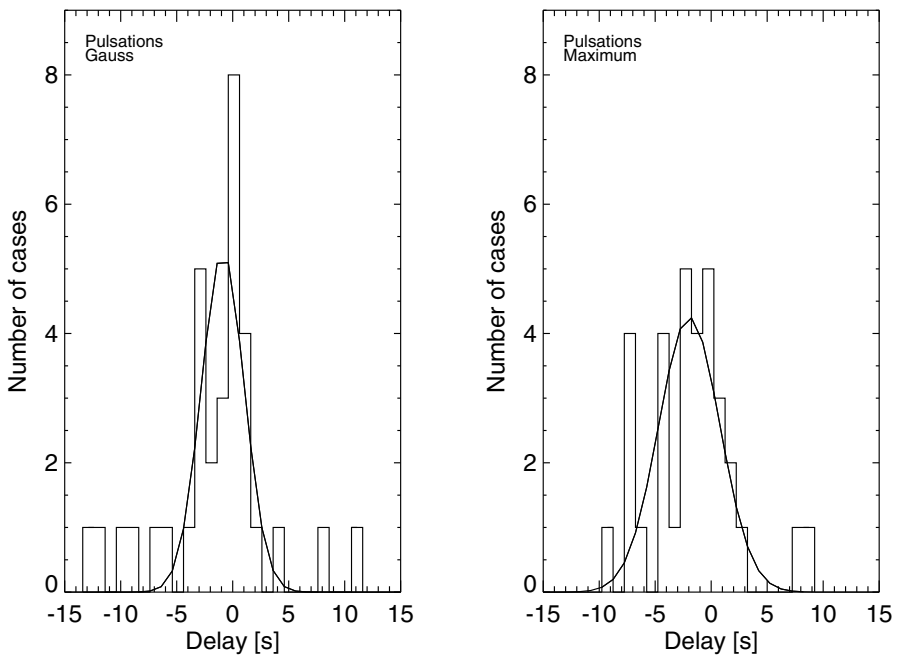

Fig. 3. The histogram of the delay for pulsations evaluated from Gauss (left column) and maximum (right column) method.
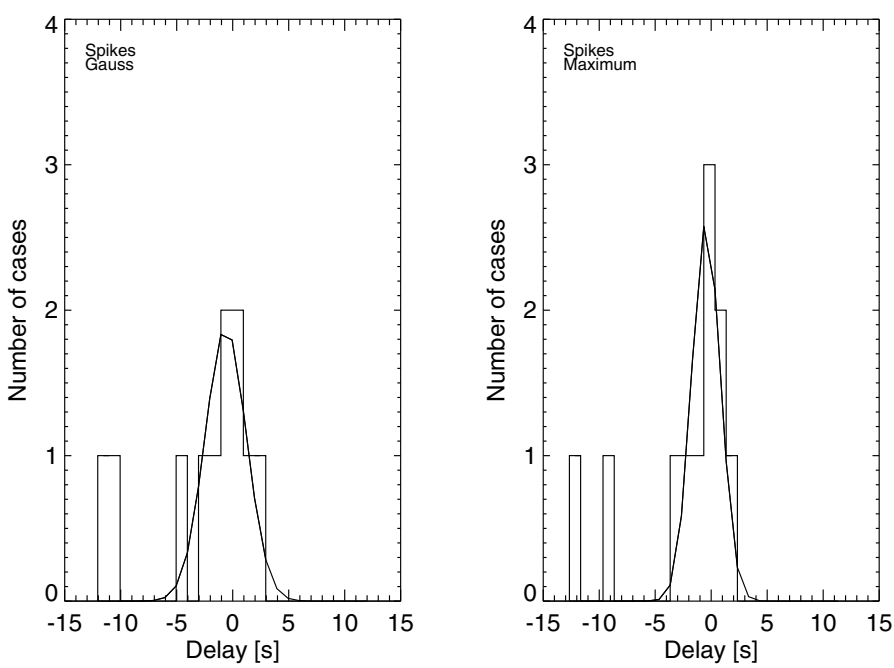

Fig. 4. The histogram of the delay for spikes evaluated from Gauss (left column) and maximum (right column) method.

The regression analysis suggests that the correlation between spikes and X-ray flux is statistically significant. The equations for this relation are the following (Gauss method):

$f_{\text {RHESSI }}=93.9-17.6 \tau$,

where $f_{\text {RHESSI }}$ is the number of RHESSI counts per second per subcollimator at peak and $\tau$ is a delay in $\mathrm{s}$.

$f_{\mathrm{GOES}}=5.2 \times 10^{-6}-3.2 \times 10^{-6} \tau$,

where $f_{\mathrm{GOES}}$ is the GOES soft X-ray flux in the $1-8 \AA$ range in units of $\mathrm{W} \mathrm{m} \mathrm{m}^{-2}$.

Other parameters were also compared with negative results for pulsations and spikes:

- no relation between delay and centre frequency;

- no relation between delay and cross-correlation coefficient;

- no relation between delay and duration;

- no relation between delay and radial position from centre of the solar disk;

- no relation between radial position from centre of the solar disk and cross-correlation coefficient.

\section{Discussion}

The distributions of delays in both types of emission and with both methods peak around zero, in particular for strong events. There are, on the other hand, also surprisingly large delays in both directions.

The results from the analysis of the delays that need to be discussed are:

- the delay of individual HXR groups or all events relative to associated decimetric pulsations and spikes, which is of the order of several seconds and can be positive or negative;

- the distribution of the delays in time that has two components: a kernel and broad wings;

- the distribution of the kernel that has a mean shift compatible with zero.

\subsection{Origin of delays}

We suggest the following interpretations:

1. Decimetric pulsation or spike emissions and X-rays occurring during a flare have two kinds of relation: $(i)$ chance associations that occur during the same flare, but have different acceleration sites, and (ii) pairs with a direct causal link and common acceleration event.

The first kind $(i)$ appears as broad wings in the delay distributions. Most events beyond some three standard deviations of the kernel belong to this population. The second kind (ii) forms the kernel of the delay distribution. The mean delay averaged over the kernel is about $-1 \mathrm{~s}$, but statistically compatible with zero.

2. The width of the kernel is produced mainly by two opposing effects: $(i)$ the delay of HXR from footpoints in the chromosphere at a larger distance than the radio sources in the corona; (ii) radio waves propagate in a plasma slower than the speed of light.

Effect (i): Benz et al. (2002) reported the location of decimetric spike sources some $20^{\prime \prime}$ to $400^{\prime \prime}$ away from the HXR source. Their observations corresponded to a frequency of $420 \mathrm{MHz}$ and $432 \mathrm{MHz}$. Allowing for projection and lower altitudes at higher frequencies, we conclude that X-rays can be delayed by about one second. The non-observation of a centre-to-limb effect may be due to its small influence relative to other effects.

Effect (ii): the delay of radio emission caused by the group velocity $v_{\text {gr }}$ being lower than the speed of light $c$ is called the group delay and is given by

$\tau=\int\left(\frac{1}{v_{\mathrm{gr}}}-\frac{1}{c}\right) \mathrm{d} s$,

integrated from the source to the observer. The group velocity is calculated from the dispersion relation of radio waves in plasmas. For $\omega \gg \Omega_{\mathrm{e}}$,

$v_{\mathrm{gr}}=\frac{\omega}{k} \approx c\left(1-\omega_{\mathrm{p}}^{2} / \omega^{2}\right)^{1 / 2}$,

where $\omega$ is the observing frequency, $\omega_{\mathrm{p}}$ is the plasma frequency, and $\Omega_{\mathrm{e}}$ is the electron gyro-frequency. Substituting Eqs. (4) into (3), one obtains to first order in $\left(\omega_{\mathrm{p}} / \omega\right)^{2}$

$$
\begin{aligned}
\tau & \approx \frac{1}{2 c \omega^{2}} \int \omega_{\mathrm{p}}^{2} \mathrm{~d} s=\frac{2 \pi \mathrm{e}^{2}}{c m_{\mathrm{e}}} \frac{1}{\omega^{2}} \int n_{\mathrm{e}} \mathrm{d} s \\
& =1.345 \times 10^{-3} v^{-2} n_{\mathrm{e}}^{0} H_{n},
\end{aligned}
$$


Table 3. Mean delay for groups of decimetric pulsations and spikes. The standard deviation of the mean value is indicated by the \pm margin.

\begin{tabular}{lcccc}
\hline \hline & \multicolumn{2}{c}{ Pulsations } & \multicolumn{2}{c}{ Spikes } \\
\hline & Gauss & Maximum & Gauss & Maximum \\
\hline Mean delay [s] & $-1.4 \pm 0.9$ & $-1.7 \pm 0.7$ & $-2.5 \pm 2.5$ & $-2.1 \pm 2.5$ \\
Gauss fit to delay distribution [s] & $-0.9 \pm 0.3$ & $-2.0 \pm 0.5$ & $-0.6 \pm 0.7$ & $-0.5 \pm 0.2$ \\
\hline
\end{tabular}
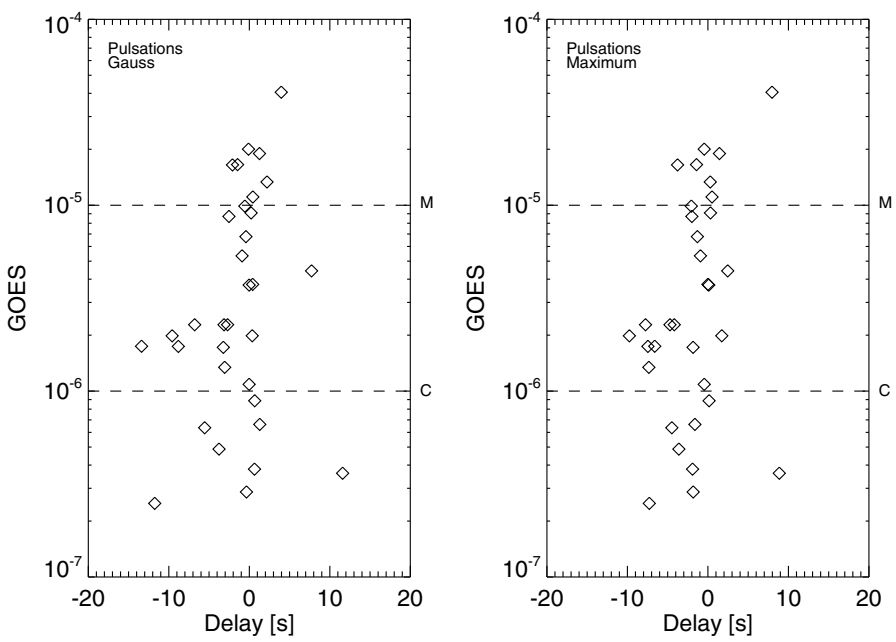

Fig. 5. The delay value of the radio emission (against HXR) connected with pulsations versus GOES magnitude of the flare.
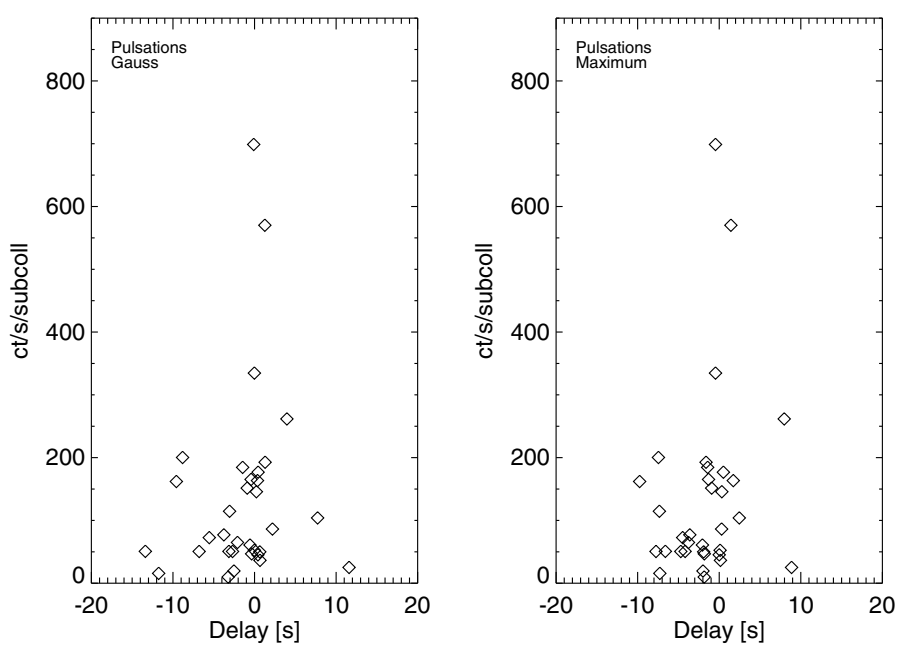

Fig. 6. The delay value of the radio emission (against HXR) connected with pulsations versus peak hard X-ray flux observed by RHESSI.

(Benz 2002), where $n_{\mathrm{e}}$ is the electron density in $\mathrm{cm}^{-3}, n_{\mathrm{e}}^{0}$ the electron density in the radio source, and $H_{n}$ the density scale height in $\mathrm{cm}$. For $v=1 \mathrm{GHz}, n_{\mathrm{e}}^{0}=10^{10} \mathrm{~cm}^{-3}$, and $H_{n}=10^{10} \mathrm{~cm}$, the group delay $\tau$ is $0.13 \mathrm{~s}$, assuming vertical propagation. It is longer for fundamental emision, and shorter for the second harmonic. Benz \& Pianezzi (1997) studied the delay beetwen different polarites for weakly polarized decimetric spikes. They found a delay of approximately $100 \mu$ s between different spike polarities. The group delay for pulsations was studied by Fleishman et al. (2002) who reported a delay of up to $20 \mathrm{~ms}$ in an exceptional event at $2.8 \mathrm{GHz}$.

We note that these delays of solar origin are much longer than the instrumental delays (Sect. 2), but insufficient to provide an adequate explanation of the observed width of the delay distribution.
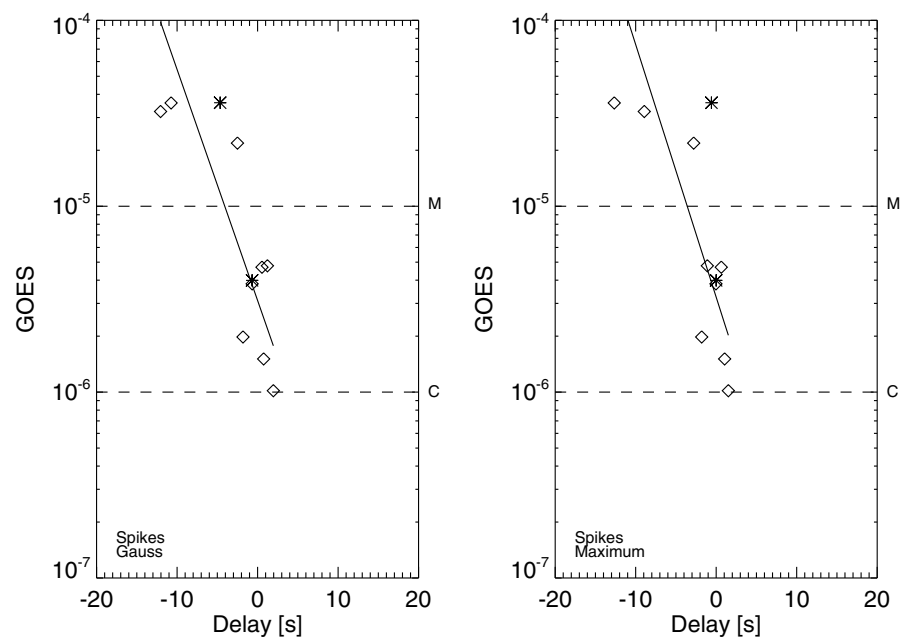

Fig. 7. The delay value of the radio emission (against HXR) connected with spikes versus GOES magnitude of the flare. Mixed events are marked with an asterisk. A straight line represents the regression relation.
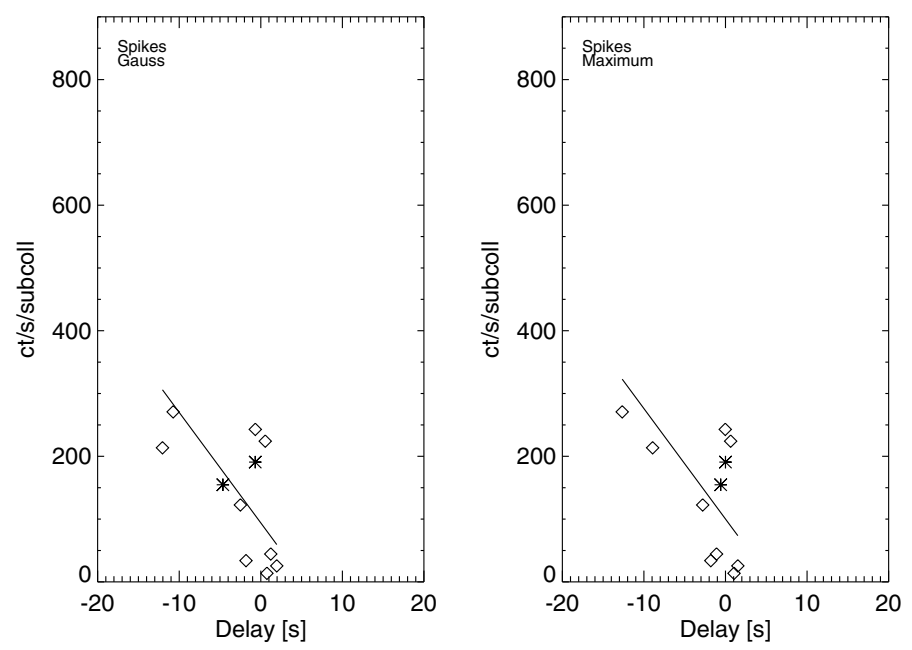

Fig. 8. The delay value of the radio emission (against HXR) connected with spikes versus peak hard X-ray flux observed by RHESSI. Mixed events are marked with an asterisk. A straight line presents the regression relation.

\subsection{Comparison with previous reports}

The mean value of the delay distribution of spikes differs from previous studies and is in-between the negative values reported by Aschwanden \& Güdel (1992) and Fárník \& Karlický (2005) for HXR and the positive value found by Fleishman et al. (2003) relative to gyrosynchrotron emission.

We first note that the distribution of delays agrees broadly with the previous reports (Sect. 1). This holds for pulsations (Arzner \& Benz 2005) as well as for spikes (Güdel et al. 1991; Aschwanden \& Güdel 1992). The relatively broad distribution of the kernel population reduces the accuracy of the mean. In addition, events from the broad population of chance coincidences 
Table 4. Parameters of the regression analysis between delay and X-ray flux.

\begin{tabular}{|c|c|c|c|c|c|c|c|c|}
\hline & \multicolumn{4}{|c|}{ Pulsations } & \multicolumn{4}{|c|}{ Spikes } \\
\hline & \multicolumn{2}{|c|}{ RHESSI } & \multicolumn{2}{|c|}{ GOES } & \multicolumn{2}{|c|}{ RHESSI } & \multicolumn{2}{|c|}{ GOES } \\
\hline & Gauss & Maximum & Gauss & Maxi & Gauss & Maximum & Gauss & Max \\
\hline Inte & 149.8 & 162.6 & $1.1 \times 10^{-5}$ & $1.13 \times 10^{-5}$ & 93.9 & 100.2 & $5.2 \times 10^{-6}$ & $5.7 \times 10^{-6}$ \\
\hline $\mathrm{S}$ & 33.4 & 38.5 & $1.8 \times 10^{-6}$ & $1.5 \times 10^{-6}$ & 28.0 & 30.8 & $1.6 \times 10^{-6}$ & $1.9 \times 10^{-6}$ \\
\hline $\mathrm{S} l$ & 9.1 & 14.8 & $3.3 \times 10^{-6}$ & $2.8>$ & -17.6 & -17.6 & $-3.2 \times 10^{-6}$ & $-3.5 \times 10^{-6}$ \\
\hline Standard deviation slope $(\Delta b)$ & 7.5 & 9.9 & $1.0 \times 10^{-6}$ & $4.3 \times 10^{-7}$ & 3.7 & 3.2 & $5.6 \times 10^{-7}$ & $8.7 \times 10^{-7}$ \\
\hline
\end{tabular}

Note: The delay of the X-rays is determined from the cross-correlation between decimetric pulsations and spikes with HXR flux (Tables 1 and 2). Linear regression is applied and the bisector reported. The function has the form $f=a+b \tau$, where $f$ is the X-ray flux (RHESSI for hard X-ray and GOES for soft X-ray) and $\tau$ is the delay using the Gauss or maximum method.

in the sample with long positive and negative delays, may dominate the mean value. Thus, the sign of the mean has a large random scatter. In the cases of Fleishman et al. (2003) and Fárník \& Karlický (2005), the number of events was 6 and 5, respectively, and the mean delay did not differ statistically from zero.

The disagreement with Aschwanden \& Güdel (1992) is more surprising. We note several possible origins: ( $i$ ) obviously the timing of modern instrument is more accurate than in previous studies, but it can hardly explain the size of the difference; (ii) the energy range considered. While Aschwanden \& Güdel (1992) based their comparison on the HXR flux from $25-50 \mathrm{keV}$, this study uses mostly lower HXR energy photons (Table 2). Because of the time-of-flight effect, low-energy electrons producing preferentially lower energy photons are delayed. However, the effect is of the order of a tenth of a seconds (Aschwanden \& Schwartz 1996) and cannot explain the difference; (iii) since we can choose the low-energy integration limit of non-thermal photons, we selected also smaller events. Furthermore, the selection of Aschwanden \& Güdel (1992) was limited to radio emissions below $1 \mathrm{GHz}$. It is unclear, however, how our more complete selection is responsible for the difference; (iv) most significant may be the different definition and selection for correlation. Aschwanden \& Güdel (1992) use all events with joint observed HXR emission, this study distinguishes between "associated" events when the radio event occurs during the enhanced HXR emission and "correlated" when both light curves, radio and X-ray emission, evolve at the same time. Only the latter were selected and only if their delay was shorter than $\pm 20 \mathrm{~s}$. The selection was not biased by the sign of the delay, but reduced the events by a factor of 3 . This allows us to distinguish more precisely between the two populations and reduces the effect of the chance coincidence population.

\section{Conclusions}

An extensive comparison between coherent flare radio-emission with non-thermal HXR has been completed using data collected during half a solar activity cycle. The data from Phoenix-2 includes 102 groups of decimetric pulsations and 25 groups of decimetric narrowband spikes well covered by good RHESSI observations. From these initially radio-selected emissions, we have found that $32 \pm 6 \%$ of the pulsations correlate in time with HXR emission, allowing for delays of up to $\pm 20 \mathrm{~s}$. In $44 \pm 24 \%$ of the decimetric spikes, there is correlated HXR emission.

The delays between radio and HXR emissions vary from group to group. The $F W H M$ width of the delay distribution is about $10.6 \mathrm{~s}$ for both types of emission. The delay distributions (Figs. 3 and 4 ) show that the population consists of a narrow kernel with FWHM of some $4.5 \mathrm{~s}$ (Gauss method) and an overpopulation of delays in the wings. We interpret these wings as chance coincidences. This concurs with the observation noted above that many decimetric pulsations and spikes are associated but only a third are correlated with HXR.

The delays by solar effects that shift the possible emission of the same population of electrons, include the longer propagation path of X-rays and the delay of the radio emission by propagation in a plasma. These two effects can explain a distribution width of about $1.5 \mathrm{~s}$. Instrumental and terrestrial effects contribute less than $0.2 \mathrm{~s}$. Thus, the observed broad distribution of $F W H M \pm 2.3 \mathrm{~s}$ can only partially be explained by delays.

The kernel of the delay distribution is nearly symmetric and centred on zero (Figs. 3 and 4). The radio emissions are delayed on average by about $1 \mathrm{~s}$, but the mean values do not differ significantly from zero, which contradicts the results of earlier work. By identifying a subgroup of decimetric emission that is strongly correlated with HXR, and has a mean delay consistent with zero, we infer that coherent radio emission is generated by particles originating from the same acceleration process as the HXR-emitting electrons. This strong radio/HXR correlation may often be undetected because of low radio emissivity or severe radio absorption.

The correlation between the delay of decimetric spikes and the SXR peak flux, previously reported, has been found to be statistically significant. However, the regression is dominated by two groups with delays of $10 \mathrm{~s}$ and more (Fig. 7). These groups may be correlated by chance (see above). Thus, the correlation is not beyond doubt and needs further confirmation.

Radiation correlating with the HXR is related to the main energy release and may provide information about the primary flare process. An occasionally good correlation may indicate a more general, but usually hidden close association. After identifying a population of decimetric pulsations and spikes correlated with HXR, the next step will be to locate these events spatially and compare them to other imaging data. With this information, it will be possible to determine whether this radio emission originates in the acceleration region or is a secondary effect caused by propagation or trapping.

Acknowledgements. We thank Christian Monstein and Hansueli Meyer for maintaining and improving the Phoenix-2 spectrometer and the RHESSI team for running the observations. We have used extensively a code developed by Kaspar Arzner. Pascal Saint-Hilaire and André Csillaghy have also substantially contributed software to this study. Help by Marina Battaglia in RHESSI software is gratefully acknowledged. The work with the Phoenix-2 solar radio spectrometers is partially funded by the Swiss National Science Foundation (grant nr. 200020-113556).

\section{References}

Arzner, K. 2004, Inverse Prob., 20, 1729

Arzner, K., \& Benz, A. O. 2005, Sol. Phys., 231, 117

Aschwanden, M. J., \& Güdel, M. 1992, ApJ, 401, 736 
Aschwanden, M. J., \& Schwartz, R. A. 1996, ApJ, 464, 974

Aschwanden, M. J., Benz, A. O., \& Kane, S. R. 1990, A\&A, 229, 206

Aschwanden, M. J., Montello, M. L., Dennis, B. R., \& Benz, A. O. 1995, ApJ, 440, 394

Benz, A. O. 1985, Sol. Phys., 96, 357

Benz, A. O. 2002, Plasma Astrophysics (Dordrecht: Kluwer)

Benz, A. O., \& Kane, S. R. 1986, Sol. Phys., 104, 179

Benz, A. O., \& Pianezzi, P. 1997, A\&A, 323, 250

Benz, A. O., Güdel, M., Isliker, H., Miszkowicz, S., \& Stehling, W. 1991, Sol. Phys., 133, 385

Benz, A. O., Csillaghy, A., \& Aschwanden, M. J. 1996, A\&A, 309, 291

Benz, A. O., Saint-Hilaire, P., \& Vilmer, N. 2002, A\&A, 383, 678

Benz, A. O., Grigis, P. C., Csillaghy, A., \& Saint-Hilaire, P. 2005, Sol. Phys., 226, 121

Cornell, M. E., Hurford, G. J., Kiplinger, A. L., \& Dennis, B. R. 1984, ApJ, 279, 875

Dạbrowski, B. P., Rudawy, P., Falewicz, R., Siarkowski, M., \& Kus, A. J. 2005, A\&A, 434, 1139

Fárník, F., \& Karlický, M. 2005, AdSpR., 35, 1799
Fleishman, G. D., \& Mel'nikov, V. F. 1998, Physics-Uspekhi, 41, 1157

Fleishman, G. D., Fu, Q. J., Huang, G. -L., Melnikov, V. F., \& Wang, M. 2002, A\&A, 385, 671

Fleishman, G. D., Gary, D. E., \& Nita, G. M. 2003, ApJ, 593, 571

Güdel, M., \& Benz, A. O. 1988, A\&AS, 75, 243

Güdel, M., Aschwanden, M. J., \& Benz, A. O. 1991, A\&A, 251, 285

Isliker, H., \& Benz, A. O. 1994, A\&AS, 104, 145

Jiřička, K., Karlický, M., \& Mészárosová, H. 2002, ESA SP-477, ed. H. SawayaLacoste, 351

Kane, S. R. 1972, Sol. Phys., 27, 174

Kane, S. R. 1981, ApJ, 247, 1113

Kane, S. R., Pick, M., \& Raoult, A. 1980, ApJ, 241L, 113

Kane, S. R., Benz, A. O., \& Treumann, R. A. 1982, ApJ, 263, 423

Kliem, B., Karlický, M., \& Benz, A. O. 2000, A\&A, 360, 715

Lin, R. P., Dennis, B. R., Hurford, G. J., et al. 2002, Sol. Phys., 210, 3

Lu, E. T., \& Petrosian, V. 1990, ApJ, 354, 735

Magdalenić, J., Zlobec, P., Messerotti, M., \& Vršnak, B. 2002, ESA SP-506, 331

Messmer, P., Benz, A. O., \& Monstein, C. 1999, Sol. Phys., 187, 335

Starr, R., Heindl, W. A., Crannell, et al. 1988, ApJ, 329, 967 